\title{
Dynamics of the PriA Helicase at the Stalled DNA Replication Forks Zhiqiang Sun ${ }^{1}$, Yaqing Wang ${ }^{1}$, Piero R. Bianco ${ }^{1}$ \& Yuri L. Lyubchenko ${ }^{1^{*}}$
}

${ }^{1}$ Department of Pharmaceutical Sciences, University of Nebraska Medical Center, Omaha, NE 68198-6025, USA;

\author{
Running title: Dynamics of PriA-DNA complexes \\ * To whom correspondence should be addressed: Yuri L. Lyubchenko: Department of \\ Pharmaceutical Sciences, College of Pharmacy, University of Nebraska Medical Center, 986025 \\ Nebraska Medical Center, Omaha, NE 68198-6025; Tel. 402-559-1971 (office); Email: \\ ylyubchenko@unmc.edu.
}



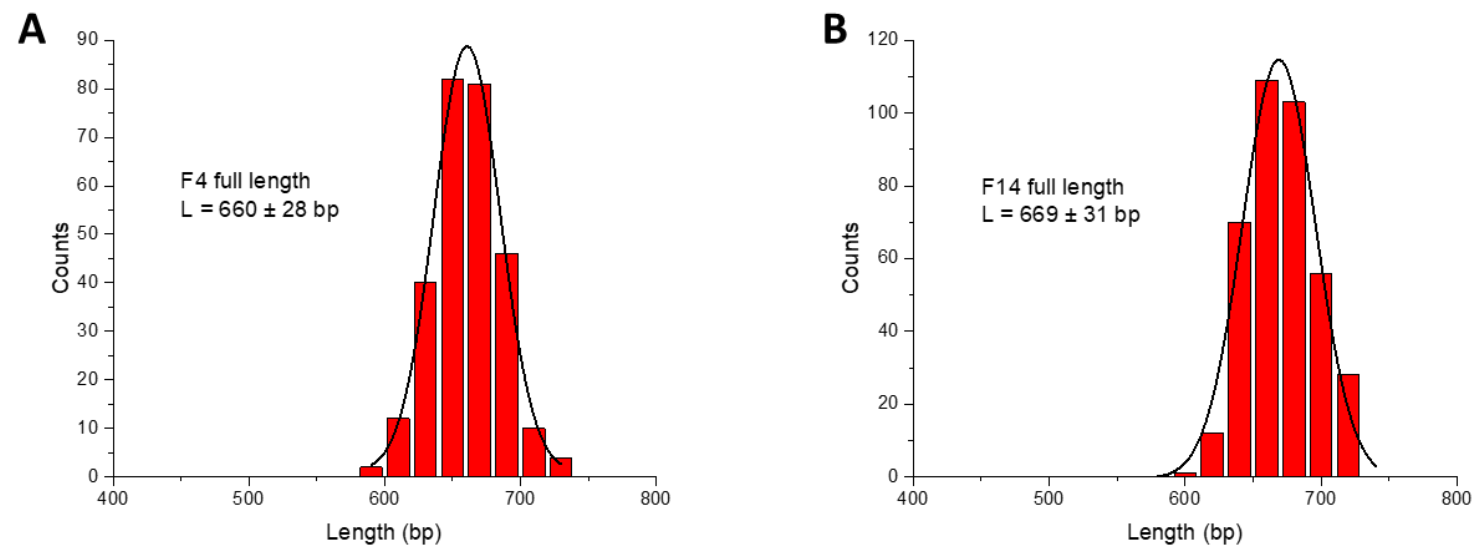

Figure S1. Contour lengths histograms of F4 (A) and F14 (B) constructs. 
A

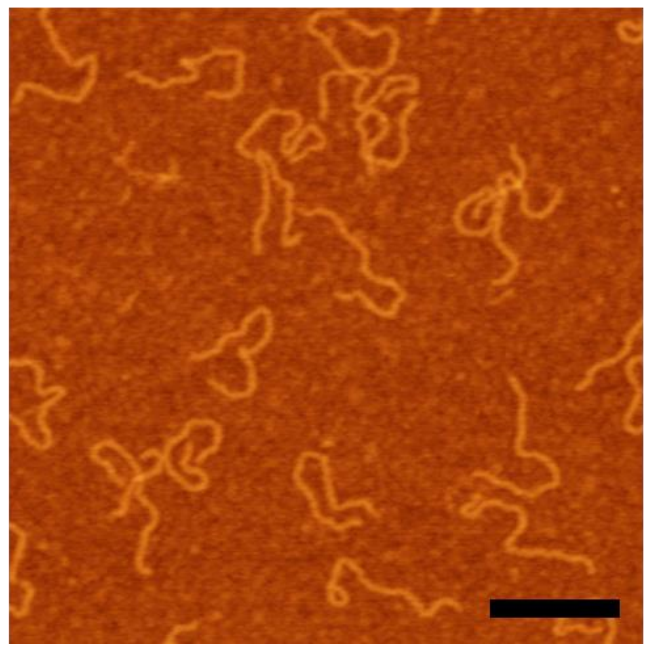

B

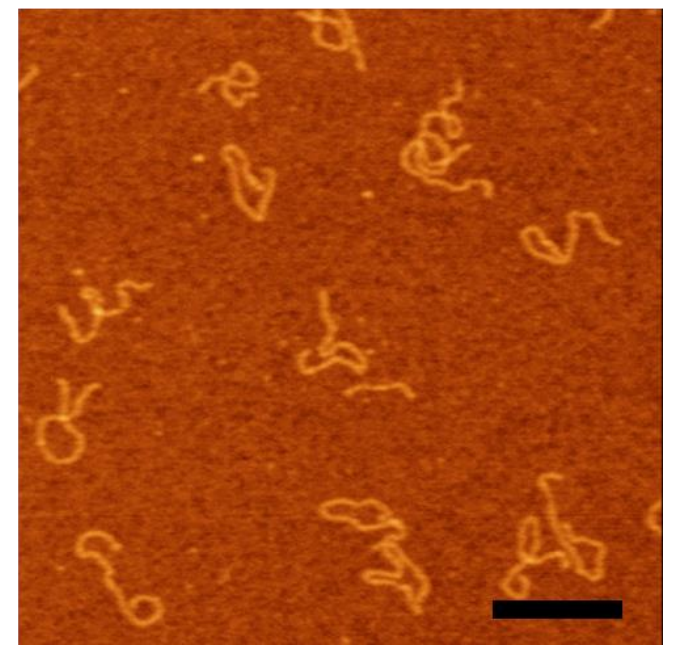

Figure S2. AFM images of complexes of PriA with double-stranded DNA in the absence (A) and presence (B) of ATP. The bar size is $200 \mathrm{~nm}$ in both images for the DNA duplex with 1036 bp. 

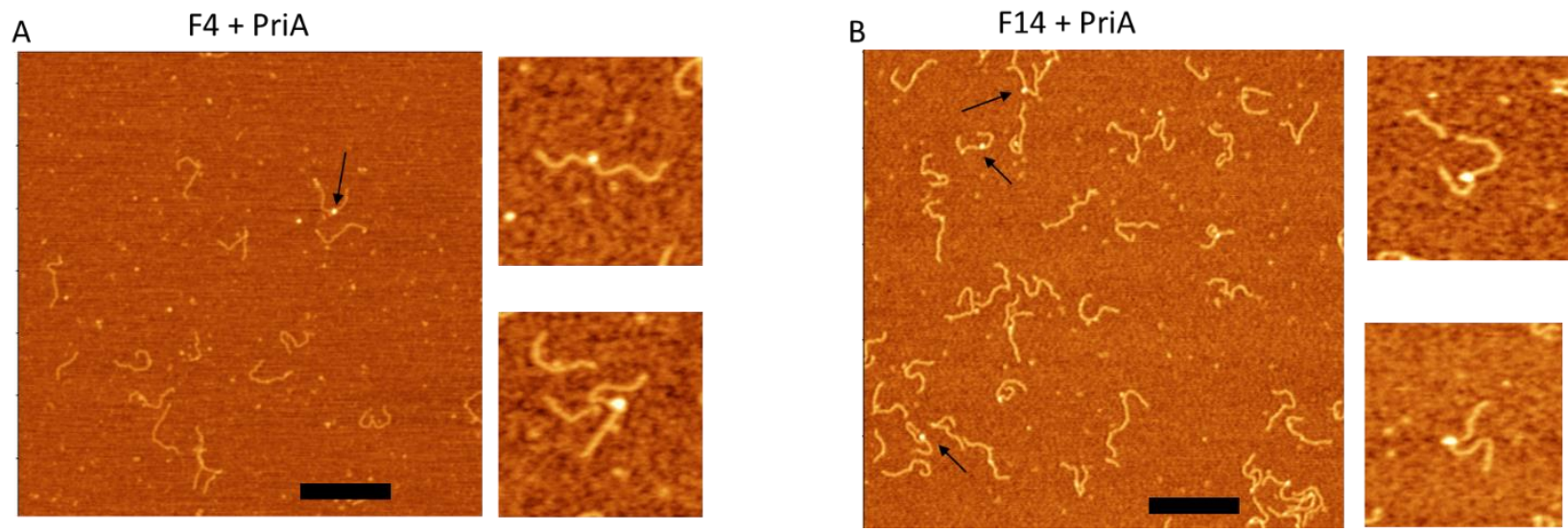

Figure S3. AFM images of PriA-fork DNA complexes in the absence of ATP. (A) Fork DNA F4 (A) and (B) for DNA F14. The bar size is $300 \mathrm{~nm}$. Arrows pointed to the protein DNA complexes. Zoomed-in images $(300 \mathrm{~nm}$ x $300 \mathrm{~nm})$ of representative complexes of PriA bound to fork DNA are shown to the right of each image. 
A

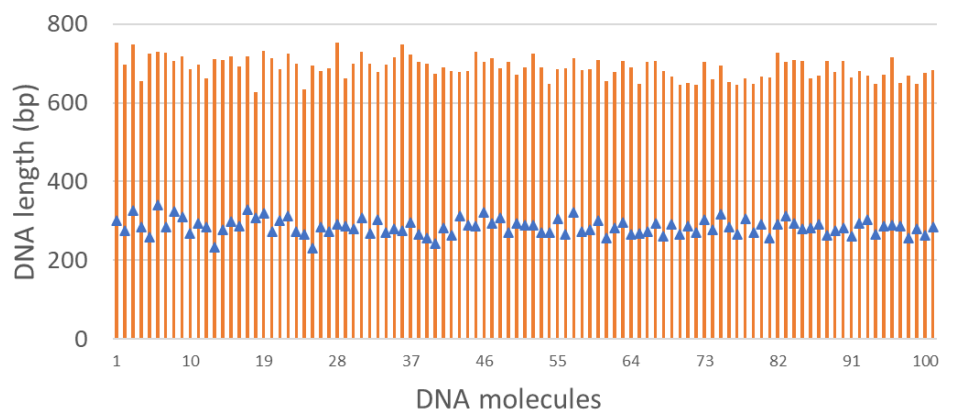

C

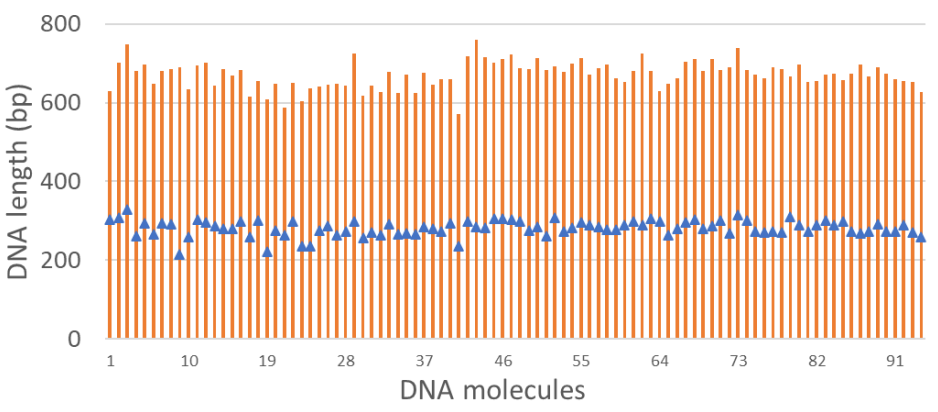

B

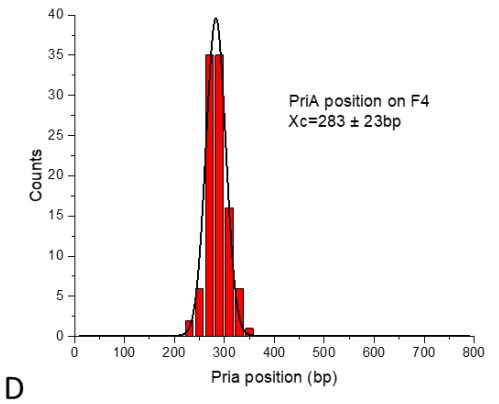

D

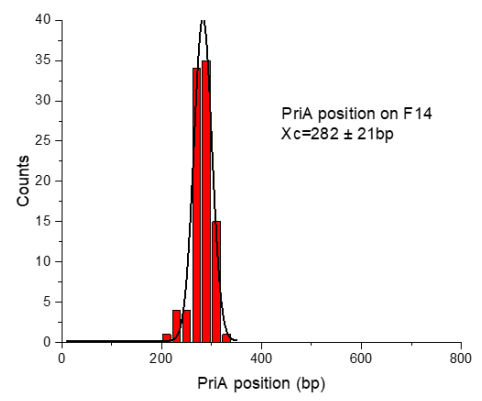

Figure S4. PriA binds to the fork but does not translocate in the absence of ATP. (A) and (C) Mapping of PriA on F4 and F14 with the absence of ATP (97 molecules for F4 and 101 for F14). DNA was aligned to the end of parental strand (short duplex) arm. The distribution of PriA relative to the end of short duplex arm distances is shown in (B) and (D). 


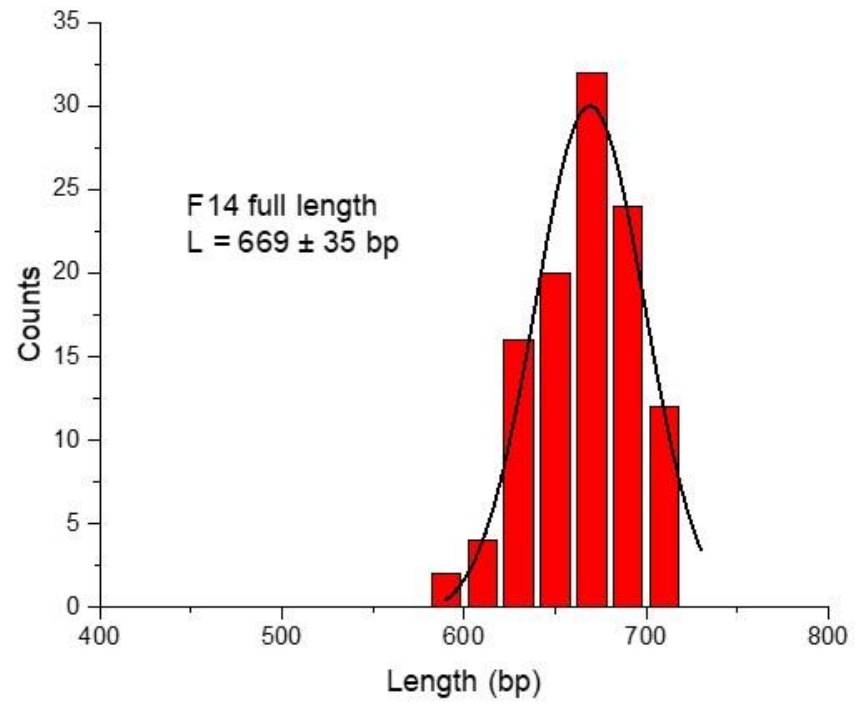

Figure S5. The full-length distribution of DNA F14 in liquid. 


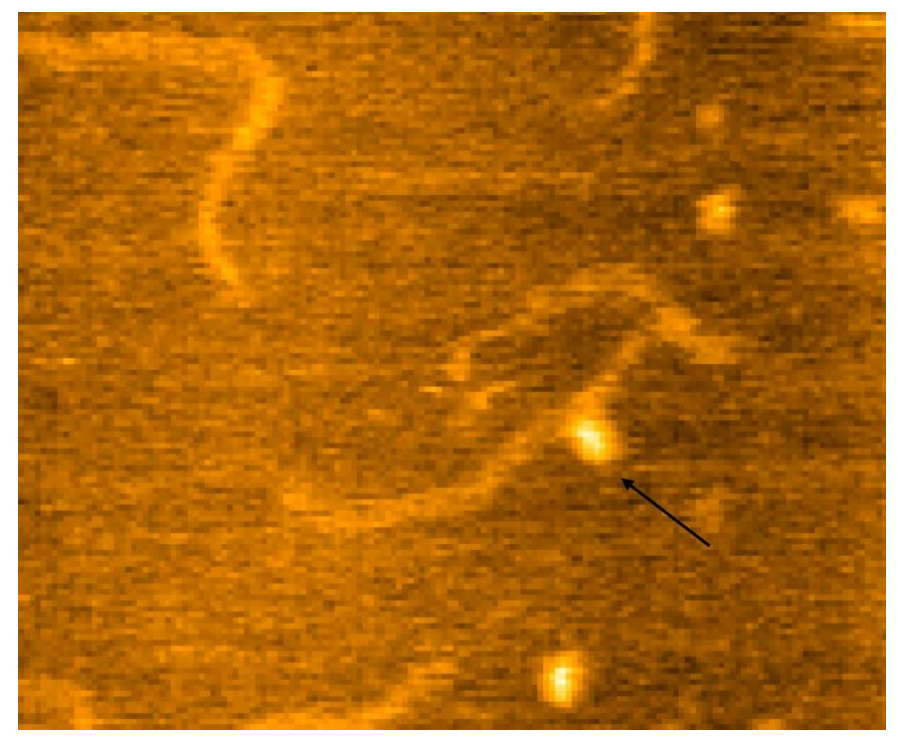

Movie S1. The data set of the time-lapse AFM experiments for PriA translocation over F14 substrate away from the fork. Arrow in the snapshot points to the PriA-F14 complex. 


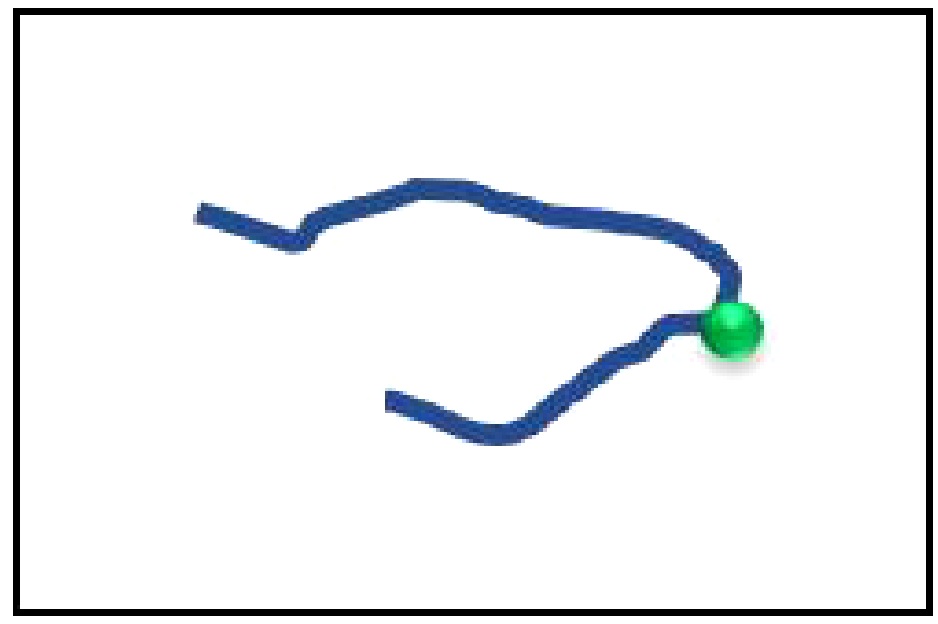

Movie S2. The movie file of assembled with traces of complexes from the frames of Supplementary movie 1 . 


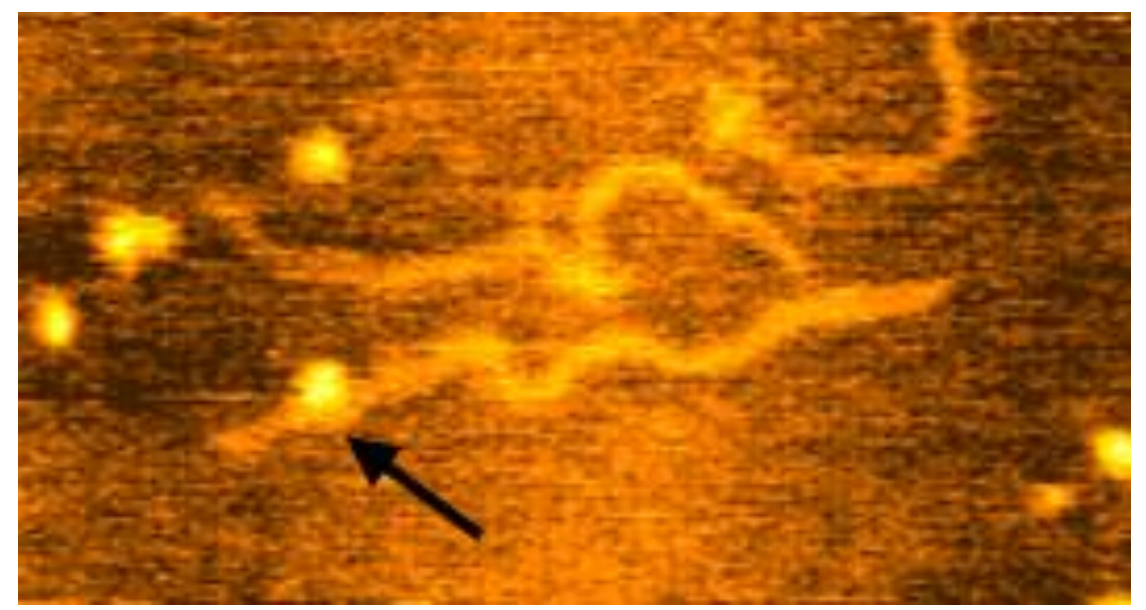

Movie S3. The data set of the time-lapse AFM experiments for PriA translocation away from the fork over F14 substrate. Arrow in the snapshot points to the PriA-F14 complex. 


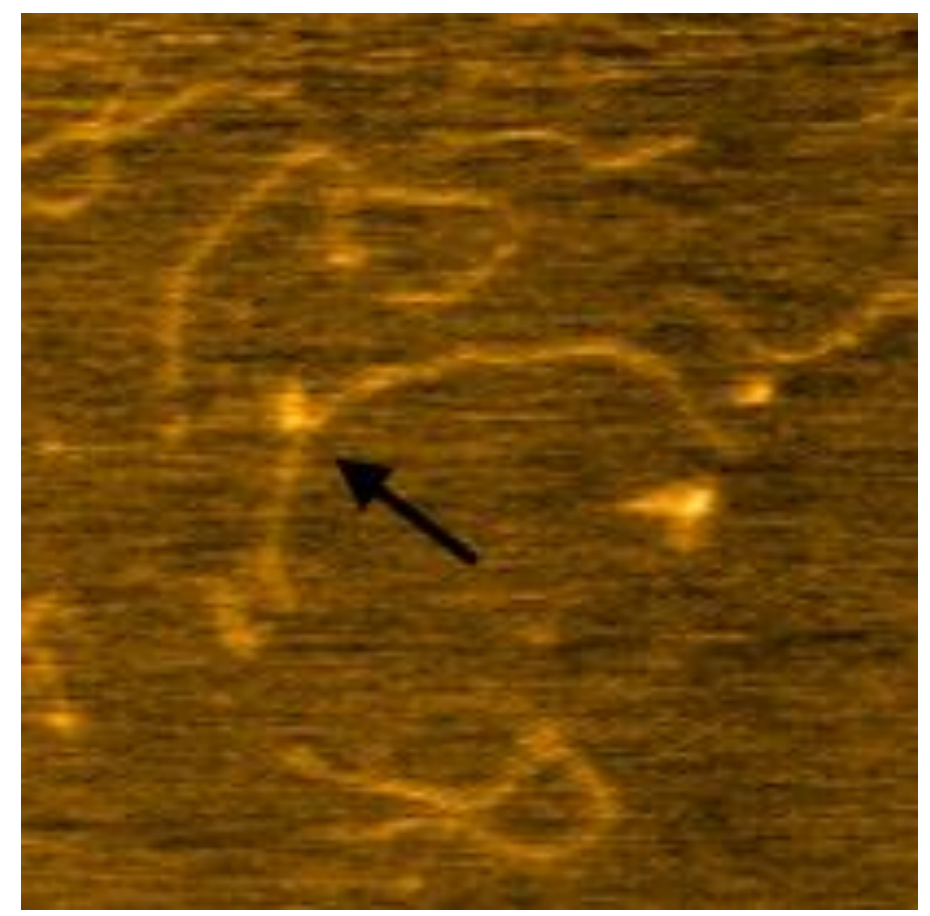

Movie S4. Another data set of the time-lapse AFM experiments for PriA translocation away from the fork over F14 substrate. Arrow in the snapshot points to the PriA-F14 complex. 


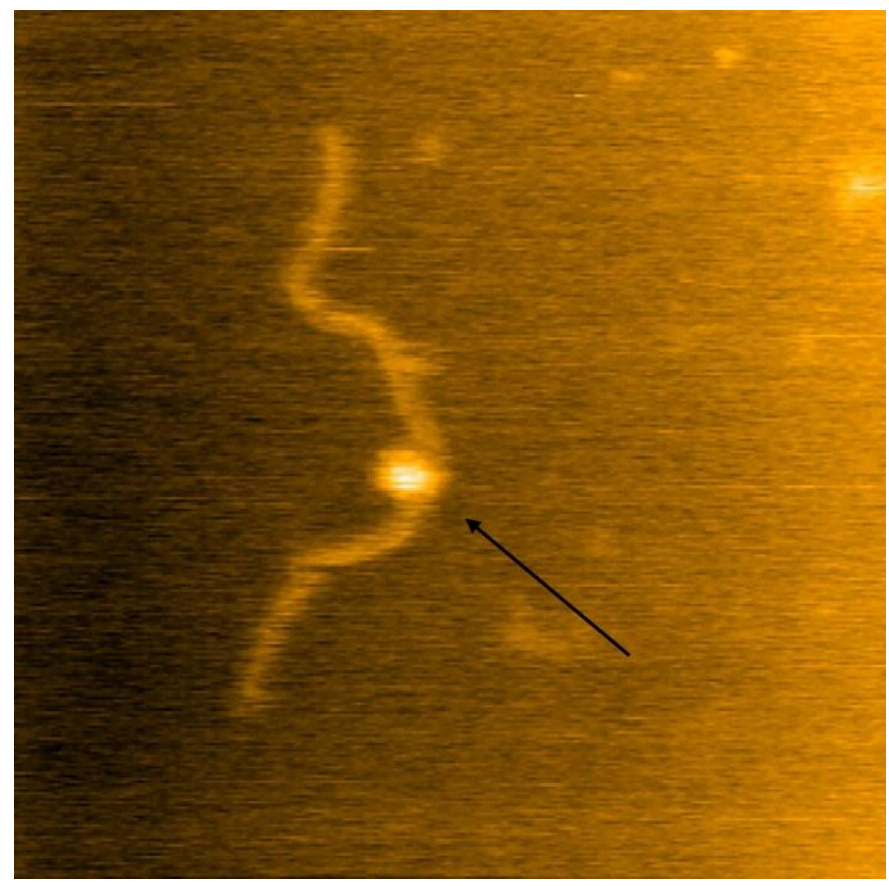

Movie S5. The dataset assembled as a movie file for PriA translocation in both directions. Arrow in the snapshot points to the PriA-F14 complex. 